\title{
Transmettre la peur et la fascination
}

Mémoire d'une interaction initiatique en pays bulongic (République de Guinée)

Bequeathing fear and fascination: the memory of initiation among the Bulongic ( Guinea)

David Berliner

\section{(2) OpenEdition} Journals

Édition électronique

URL : http://journals.openedition.org/span/774

DOI : $10.4000 /$ span.774

ISSN : 2268-1558

Éditeur

École pratique des hautes études. Sciences humaines

Édition imprimée

Date de publication : 1 décembre 2008

Pagination : 105-131

ISSN : 0294-7080

Référence électronique

David Berliner, «Transmettre la peur et la fascination », Systèmes de pensée en Afrique noire [En ligne], 18 | 2008, mis en ligne le 02 juillet 2013, consulté le 20 avril 2019. URL : http://

journals.openedition.org/span/774; DOI : 10.4000/span.774 


\section{Transmettre la peur et la fascination}

Mémoire d'une interaction initiatique en pays

bulongic (République de Guinée)

\section{David Berliner*}

Professeur

Université Libre de Bruxelles

La question de la transmission ne se pose que pour un être fini... ${ }^{1}$

\section{Introduction}

Comment se transmettent des idées, des pratiques et des émotions? Comment expliquer la longévité de certaines d'entre elles? Concrètement, s'interroge Debray, « comment se fait-il qu'il subsiste aujourd'hui en Occident, deux mille ans après Jésus, quelque chose comme du christianisme; plus d'un siècle après la mort de Marx ou de Darwin, du marxisme ou du darwinisme; plus de quinze ans après la mort de Lacan, du lacanisme, etc. ? " Autant de questions qui suscitent aujourd'hui un intérêt particulier en anthropologie, mais qui préoccupaient déjà les pères de la discipline ${ }^{3}$. Pour aborder les questions de persistance et de transmission culturelles, des anthropologues ont récemment mis l'accent sur la nécessaire collaboration de notre discipline avec les sciences cognitives ${ }^{4}$. Au croisement de l'anthropologie religieuse et des dernières avancées dans le domaine de la cognition, la plupart de ces auteurs cherchent à montrer comment l'architecture cognitive humaine contribue à la génération, la transmission et la distribution des idées et pratiques religieuses. Boyer a, par exemple, montré pourquoi certaines hypothèses religieuses seraient plus transmissibles que d'autres ${ }^{5}$, tandis que McCauley et
*Que soient remerciés tous les Monchonais que je ne peux citer ici mais qui m'ont tant aidé (en particulier Hervé Camara, El Hadj Momodoubé Bangoura, El Hadj Abdou

Bangoura,

Papa Bangoura).

Je tiens aussi à

remercier

Philippe Jespers,

Ramon Sarro,

David Parkin,

Peter Geschiere,

Filip De Boeck, Pierre

Petit et, enfin,

Michael Houseman

dont les travaux sont

une source majeure

d'inspiration.

${ }^{1}$ Soual, 2003 : 100.

2 Debray, 1997 : 25.

${ }^{3}$ Berliner \& Sarro,

2007.

${ }^{4}$ Bloch, 1998;

Boyer, 1997, 2001;

Whitehouse, 2001 ;

Hojbjerg, 2002.

${ }^{5}$ Boyer, 2001. 
${ }^{6}$ McCauley \& Lawson, 2002.

7 Houseman, 2004 ; Berliner \& Sarro, 2007.

${ }^{8}$ Simmel, 1996.

9 Bellman, 1984;

Zempléni, 1976, 1996;

Houseman, 1993,

2002; Herdt, 2003:

Bonhomme, 2006.

${ }^{10}$ Bellman, 1984 : 140.

11 Shaw, 1997 : 44.
Lawson, dont l'ambition générale est de fonder une théorie cognitive du rituel, entendent dé-sémiotiser le rite et s'interrogent sur l'existence de propriétés formelles intrinsèques au rite qui le rendraient meilleur à transmettre ${ }^{6}$. Je pense toutefois qu'à insister sur les processus cognitifs de la transmission, à toujours donner la préséance aux opérations psychologiques des acteurs qui permettraient d'expliquer les mécanismes dits " réels " de la transmission, ces approches mentalistes passent trop souvent à côté du jeu complexe des forces sociales que la transmission et l'apprentissage culturels mettent en jeu ${ }^{7}$. Comment des représentations, des pratiques, des émotions religieuses sont-elles transmises et apprises dans les situations sociales concrètes? Qui transmet quoi et comment? Dans quels réseaux de transmission et d'apprentissage et dans quelles formes d'organisation tel héritage religieux s'est-il constitué? Dans cet article, je vais précisément me préoccuper du niveau de l'interaction sociale et mettre l'accent sur la persistance d'une relation toute particulière qui existe entre les partenaires de l'interaction.

L'on sait, depuis les contributions de Simmel ${ }^{8}$, enrichies par celles de Bellman, Zempléni, Houseman, Herdt et Bonhomme ${ }^{9}$, que, bien que doté d'un contenu, le secret est avant tout chose relationnelle, "an accomplished interactionnal phenomenon ${ }^{10}$ ", qui opère une distinction entre les détenteurs du secret et ceux qui en sont exclus. Dans nombre de sociétés africaines, l'existence d'associations d'hommes initiés, dérivant "their powers from their control of secret knowledge "11 ", met en jeu ce genre de structures interactives dynamiques articulant différentes perspectives. L'existence du secret trace, en effet, de nombreuses lignes de partage entre ceux qui savent et ceux qui ne savent pas, ceux qui peuvent et ceux qui ne peuvent pas, ceux qui, comme dit Zempléni, "sécrètent " et ceux qui ne « sécrètent " pas, bref entre ceux qui participent du secret et ceux qui n'en participent pas.

Connus dans la littérature ethnographique sous le nom de Baga Forè que leur donnent leurs voisins Susu, 
les Bulongic vivent sur les rives du Rio Kapatchez (GuinéeConakry) ${ }^{12}$. Ils ont été de grands sculpteurs de masques et de puissants ritualistes connus pour leurs sociétés d'initiation masculines. Un processus d'islamisation relativement tardif a mis fin à ces pratiques initiatiques. En effet, depuis le début du $\mathrm{xx}^{\mathrm{e}}$ siècle, les Bulongic ont fini par embrasser la religion musulmane, sur fond de destruction d'objets et de forêts sacrées. Dans cet article, je voudrais montrer que, dans le village bulongic de Monchon, persiste, sans objet ni rituel visible, une "structure relationnelle dynamique ${ }^{13}$ " liée au passé initiatique pré-islamique. J'insisterai sur la relation qui existe entre les vieux qui ont été initiés en 1937 et en 1954, et les jeunes générations qui ne le seront jamais, et montrerai comment dans le tissu de cette interaction, en l'absence de rite, se déploie une "épistemologie du secret ${ }^{14}$ " à forte charge émotionnelle, qui oscille entre la peur et la fascination.

\section{L'initiation à Monchon}

En 1954, Denise Paulme est la première à mettre en relief la présence d'une structure sociale dualiste à Monchon. Le village se répartissait entre la moitié masculine Camara - qui passait pour abriter les premiers habitants de Monchon - et la moitié féminine Bangoura. Cette " opposition complémentaire " s'illustrait lors de la circoncision des jeunes garçons sous la forme d'un combat entre deux masques serpentiformes, "poutres peintes et décorées, hautes chacune d'environ six mètres, qui surgiss[ai]ent de la rizière pour s'élancer l'une contre l'autre dans la foule ${ }^{15}$ ". Car chaque moitié possédait son masque circonciseur et sa forêt sacrée. Côté Camara, le masque masculin Mosolo Kombo était conservé dans Amancongopun, la forêt du quartier Kitala; le masque féminin Mosolo Sangaran de la moitié Bangoura était gardé dans la forêt Kumbumpun, quartier Kiyaye. Les ethnologues et les historiens de l'art ont publié d'abondantes représentations de ces deux masques, en le désignant improprement par le mot susu bansonyi. Le terme générique qui le désigne à Monchon est amancong,

\author{
12 Coincés entre \\ les Baga Sitem au nord \\ et les Baga Kakissa au \\ sud, les Bulongic sont \\ approximativement \\ six mille. Les princi. \\ paux villages bulongic \\ sont Kaktè, Kiffinda, \\ Monchon, Mintani, \\ Mambacho, Bongolon et \\ Yamponi (voir Paulme, \\ 1956, 1957; Berliner, \\ 2002). Les données \\ exposées ici ont été \\ recueillies entre 1998 \\ et 2001 , lors de séjours \\ effectués principale. \\ ment dans le village de \\ Monchon, et complétés \\ par de brèves recher. \\ ches comparatives en \\ pays landuma, baga \\ sitem, kakisa et koba. \\ 13 Houseman, 1993; \\ Houseman \& Severi, \\ 1994.
14 Barth, 1975.
Nombreux sont
les anthropologues à
s'être exprimés sur
la difficulté à travailler
dans un environnement
saturé de secrets. Je
tiens à rappeler qu'il
n'a jamais été question
mais plutôt d'essayer
d'en comprendre les
mécanismes sociaux.
Dans tous les cas,
comme on va le voir
les vieux initiés bulongic
ont mis en place des
techniques de dissimu.
lation hautement
sophistiquées destinées
à protéger certains de
leurs domaines réservés
de savoir.
15 Paulme, 1956 : 106. \\ de dévoiler des secrets,
}


16 Lamp, 1996;

Sarro, 1999; Curtis, 1996; Berliner, 2004.

${ }^{17}$ Sinayoko, $1937: 221$.

${ }^{18}$ Paulme, $1958: 412$. mot bulongic dont bansonyi est peut-être un lointain dérivé et dont on trouve des variantes dialectales dans les divers noms qui désignent les masques circonciseurs chez les autres Baga ${ }^{16}$.

Choses de moitié, bien que détenues chacune par un patrilignage en particulier, les puissances représentées par ces masques regroupaient tous les hommes circoncis de plusieurs villages parents, organisés en un réseau initiatique complexe. Alors qu'à Monchon, Sangaran était porté uniquement lors de la circoncision, Mossolo Kombo intervenait en de multiples lieux et contextes. En 1937, alors enseignant dans ce village, S. Sinayoko avait déjà décrit certains des contextes d'action de Mossolo Kombo (qu'il appelle le Mansonyi) :

Il était le fétiche à qui incombe la garde perpétuelle du village. Lors d'une naissance, c'est devant la pierre dédiée à son emblème qu'à genoux la maman se confond en remerciements et recommande son enfant à sa protection. C'est pour lui qu'est recueilli, à la mort d'un patriarche, dans le crâne sacré le sang d'un taureau noir offert aux mânes des ancêtres. Certaines nuits, il sort clandestinement dans le village et malheur au sorcier qui fait sa rencontre; dès le jour, il se confesse et expire aussitôt. [...] La sécheresse vient-elle à menacer les cultures, c'est dans la petite hutte érigée en l'honneur de son culte que l'assemblée des vieux hommes implore son secours pour faire tomber l'eau. S'agit-il d'élucider quelque question d'ordre social, il faut aller délibérer dans le bois sacré où le fétiche a choisi son séjour ${ }^{17}$.

Au mois de décembre, Mossolo Kombo visitait la plaine pour contrôler les bonnes et les mauvaises récoltes. Il empêchait par sa présence qu'une certaine jalousie ne se développe entre les cultivateurs. Véritable instance morale selon les vieux initiés, il interdisait le gaspillage, le vol, le mensonge, les calomnies. À cet égard, l'on jurait sur Mossolo Kombo, ce qui signifiait la mort en cas de parjure. Il ne fait pas de doute que ce grand masque, «insigne de la société des hommes, protecteur des jeunes gens durant la retraite d'initiation, [...] talisman bénéfique par excellence $^{18}$ ", ait joué autrefois un rôle rituel, moral et social de première importance. De part en part, il dominait la vie des hommes, depuis l'acquisition de la masculinité à travers 
l'initiation jusqu'à la punition des infractions, en passant par la subordination des femmes aux hommes.

L'on ne peut situer l'inscription de Mossolo Kombo et de Sangaran dans la société bulongic pré-islamique sans référer à l'initiation. Les gens de la moitié Camara étaient obligatoirement initiés à Mossolo Kombo, tandis que les Bangoura suivaient l'initiation de Sangaran. Existait un fort sentiment d'appartenance à une moitié rituelle ou à l'autre, qui se traduisait par l'identification à l'entité initiatique elle-même: "Moi, je suis Mossolo Kombo" ou "Moi, je suis Sangaran", s'exclament aujourd'hui encore de vieux Bulongic. Ces entités initiatiques opéraient la transformation des jeunes garçons non-circoncis, les birakurung, en hommes circoncis, les bilgbili. Lors de l'initiation suivante, abandonnant leur statut de bilgbili, ces derniers devenaient bilgbilifang, initiateurs, et ce, après le rite appelé amancong wala (littéralement "attraper Amancong»). Le matin du début de l'initiation, quatre des nouveaux initiés étaient circoncis avant les autres et désignés pour « attraper Amancong ». Désignés de par leur patrilignage d'origine bien avant le déroulement de ce rite, ces quatre premiers circoncis étaient les futurs responsables de la forêt sacrée. Cette épreuve consistait pour les quatre élus à courir entre deux rangées de plus de cent personnes et à subir, le temps de cette course, un déferlement de coups. Au bout de la rangée, se trouvait Mossolo Kombo. Le but était, pour le bilgbili, d'attraper une petite corne située aux pieds du masque. Après amancong wala, sous la responsabilité des tout nouveaux bilgbilifang, la circoncision pouvait alors commencer.

L'initiation était un événement social et rituel de première grandeur dont l'organisation nécessitait plusieurs mois de préparation. Elle était l'occasion de très nombreux sacrifices de bøufs, d'une consommation extraordinaire de vin de palme et de riz. Les deux initiations, à Sangaran et à Mossolo Kombo, avaient lieu au même moment, théoriquement tous les 24 ans $^{19}$. La circoncision était précédée d'une période de six mois où les vieux "fermaient la terre " (kcang bong). Fixé collégialement par les vieux des différents 
20 Sinayoko, 1937 : 222. villages, ce laps de temps pré-initiatique était caractérisé par une série d'interdits pesant sur les villageois ("Pas de danse, de cri, de bruit, de vol, d'adultère pendant kcang bong ", selon l'un de mes interlocuteurs). Lors des deux dernières initiations datées, en 1937 et en 1954, après la circoncision (biliwong) qui marquait le début de la réclusion initiatique, les jeunes garçons passèrent six mois à l'écart du village, isolés des femmes. Sous la direction de leurs bilgbilifang, tous y apprirent des chants et danses et subirent, en présence des masques et d'autres entités, des épreuves destinées à mesurer leur maturité physique et morale. À en croire aujourd'hui les vieux, les jeunes circoncis étaient régulièrement battus, insultés, humiliés. Affamés, ils devaient marcher sous le soleil durant de longues heures à travers la mangrove. Mais surtout, ils étaient exposés à Mossolo Kombo « rouge comme le sang ", à Sangaran « dont les yeux brillaient comme ceux d'un chat " et aux bèn bé kiyitun (littéralement « ceux qui sont dans la mer »), des entités marines (invisibles mais sonores) accompagnant les masques circonciseurs. Pour témoigner de la violence sociale et physique de l'initiation, une violence décrite comme un ingrédient indispensable à cette expérience, un vieil initié raconte :

Pendant six mois, les femmes ne voient plus les circoncis. Dans la forêt, on ne mange pas dans des calebasses, pas de sel, pas de sauce. Seulement dans des paniers avec du riz et quelques poissons grillés. [...] Là-bas, on te frappe bien. [...] Souvent un enfant pouvait mourir dans la forêt. Si un enfant mourait, le papa était au courant, mais les mamans ne pouvaient pas savoir...

Le début et la fin de l'initiation étaient marqués par des danses, chants et apparitions publiques des masques d'initiation. Sinayoko décrit l'une de ces performances :

Une formidable décharge de fusils ébranle le village. [...] Enfin, le masque [...] émerge de la verdure couvrant la plus profonde partie du marigot. Il vacille un moment et glisse avec une rapidité prodigieuse [...]; il pointe vers le village, entouré d'un cercle épais formé par tous les jeunes gens initiés. Seul le haut du masque est visible; [...] Les suivants règlent leur pas sur le mouvement du fétiche, chantent et dansent en rond avec des palmes dans les mains. Le "Mansonyi » entre au village dans un vacarme infernal; il le traverse et va se réfugier dans le bois sacré. ${ }^{20}$ 
À la fin de l'initiation, les bilgbili rentraient au village et étaient introduits dans la vie des hommes initiés. Comme cela a souvent été décrit, leur attitude vis-à-vis de leurs mères et de leurs sours devait se transformer ${ }^{21}$. Il leur était désormais donné de participer à des activités dont ils étaient jusqu'alors écartés, découvrant de nouvelles dimensions du système des masques.

\section{Fiction initiatique et épistémologie du secret}

Irruptions fortuites et spontanées de l'invisible, parfois manifestations sonores, Mossolo Kombo et Sangaran, dont on raconte qu'ils ont été "trouvés dans la mer", étaient des cincalleng (des "génies $\left.{ }^{22} »\right)$, des puissances autonomes, indépendantes, terrifiantes, qui surgissaient de la mangrove et envahissaient l'espace du village. En témoigne le rite qui, au début de chaque nouvelle circoncision, consistait pour les hommes initiés "à aller chercher les masques à la mer " et à revenir au village le corps couvert de poto-poto (boue argileuse de mangrove).

À Monchon, les masques étaient « en eux-mêmes des entités spirituelles", capables "de se manifester spontanément dans l'ordre du visible ${ }^{23}$." Enchanteurs, ils produisaient un effet : celui de faire "croire " à leur efficacité, quelle qu'elle soit (lutter contre les instances malveillantes, protéger les jeunes initiés, garantir l'intégrité du village, etc.); telle était, en tout cas, la fiction produite par les hommes et leurs masques.

Dans un article devenu célèbre, Smith a finement décrit ce processus d'enchantement, de "simulation initiatique ". Sorte de "piège à pensée ", il fonctionne d'une part au niveau des initiés aux secrets, qui doivent incarner, sous les masques ou par le son d'instruments, la présence sensible d'entités invisibles, et « qui se sentent et se disent investis d'une mission sacrée qui les met en contact plus étroit avec les puissances invisibles ${ }^{24}$." D'autre part, au niveau du public des non-initiés, tenu à l'écart des dessous de la mise en scène, qui se laisse idéalement prendre au piège

\footnotetext{
${ }^{21}$ La Fontaine, 1985.

${ }^{22}$ Le terme callè est traduit par "génie » (ou « diable » par les Bulongic qui ont appris le français auprès des missionnaires catholiques). Seuls certains hommes et femmes ont la réputa. tion de pouvoir les voir et d'entrer en contact avec eux.

${ }^{23}$ Adler, $1998: 175$.

${ }^{24}$ Smith, $1984: 5$.
} 
${ }^{25}$ Coquet, $1999: 25$.

26 Ibidem.

27 En pays bulongic, le terme kutum sert à désigner l'ensemble des pratiques, représentations et institutions religieuses qui préexistaient à l'implantation de la religion musulmane. et qui croit ou du moins fait mine de croire qu'il s'agit de manifestations d'entités immatérielles. De fait, si la fiction des masques repose sur une série d'hypothèses relatives à la présence d'entités dans le monde et aux modalités de leur présence, elle est également inscrite dans une configuration sociale qui articule une pluralité de discours et de catégories sociales, de comportements et d'interdits. Pour comprendre ce phénomène, il faut prendre en compte le « jeu continu, et vertigineux, d'interprétations, de gloses et d'hypothèses $^{25}$." Le masque "ne fait pas sens de la même manière pour son auteur, son propriétaire, l'initié, le non-initié, le simple passant, [...] l'autochtone et l'étranger, etc. ${ }^{26}$ ".

À Monchon, une ligne de partage très nette existait entre les hommes initiés, qui simulaient la présence sensible de masques, et les non-initiés. Mossolo Kombo et Sangaran appartenaient à la catégorie tolum (molumil au pluriel), une notion particulièrement difficile à traduire mais qui témoigne du rapport fondamental que les Bulongic entretiennent à l'égard du secret. Tolum réfère à ces secrets qui confèrent à ceux qui les détiennent malignité et efficacité rituelle extraordinaire. Secrets puissants et hautement protégés, dont peut dépendre la survie d'une famille ou d'un village, les molumil sont réservés à certains individus de la société et, corollairement, frappés de multiples interdictions. Ceux qui possèdent des molumil doivent être en contact avec les cincalleng (les génies) et sont susceptibles d'utiliser leur connaissance avec de mauvaises intentions. Pour une bonne part, le concept désigne la forêt sacrée, tout ce qui s'y déroulait et ne pouvait être dit au village devant les non-initiés (c'est-à-dire les femmes, les jeunes garçons non-circoncis et les étrangers susu). De fait, la notion signalait, notamment, l'existence des secrets de l'initiation et le système de prohibitions qui entourait les pratiques initiatiques. À entendre mes interlocuteurs âgés, tolum était une puissante catégorie d'exclusion dont la force illocutoire inspirait la peur et la fascination pour tous ceux qui n'en étaient pas. Comme me le rappelait un vieil homme, " ceux qui ont fait la coutume ${ }^{27}$ comme nous, on a des molumil que l'on ne peut même pas dire à nos mamans. Nous, les Bulongic, nous sommes les 
hommes du secret. On égorgeait les gens qui révélaient les secrets. C'est ça, tolum!»

En effet, quiconque n'était pas initié au secret des masques était soumis à une série de règles l'empêchant soit de voir les masques, soit de s'en approcher. À chaque apparition de Mossolo Kombo et Sangaran, les femmes de Monchon s'enfuyaient et devaient rester terrées dans leurs cases. Certes, avant 1954, les femmes se réunissaient déjà dans le cadre de leur société secrète kèkè et avaient leurs propres rites. Mais, à entendre mes interlocuteurs masculins et féminins aujourd'hui, elles n'en étaient pas moins subordonnées aux pratiques rituelles masculines, toujours exclues des espaces et contextes rituels dominés par les hommes, bien qu'elles aient aussi joué le rôle d' " autre noninitiable " déjà défini par $\mathrm{Smith}^{28}$. Le même régime s'appliquait à tous les non-initiés, qui ne pouvaient en aucun cas entrer dans les forêts sacrées, mais qui avaient part à des propos et des rumeurs qui venaient renforcer la fiction des initiés. Pour eux, les molumil initiatiques étaient créateurs d'interdits et d'obligations : se cacher, ne pas sortir, ne pas voir, ne pas être vu, ne pas poser de questions, ne pas s'approcher, manifester du respect, saluer, parler, répondre ou danser ${ }^{29}$. Les non-initiés avaient une conscience aiguë du danger de spéculer ou de vouloir s'approcher de la connaissance secrète de leurs pères ${ }^{30}$. Comme on le verra plus loin, l'absence de questions faisait partie de l'épistémologie même du secret.

De leur côté, les hommes initiés, détenteurs des molumil, produisaient volontairement du secret, l'exhibant devant ses destinataires sans pour autant le communiquer, ni le révéler, tout en intimidant les non-initiés. Il fallait savoir embrouiller quand cela était nécessaire et utiliser des langages incompréhensibles (les initiés avaient leur propre langue secrète) ou des appellations inconnues toujours destinées à renforcer l'existence du secret. Par exemple, Mossolo Kombo avait plusieurs surnoms: Utem Kombo, Ncaman, Tongbonkolong, tous destinés à «tromper les non-circoncis ». Il fallait aussi des espaces reculés et cachés, des forêts sacrées. Mais surtout, la fiction initiatique avait

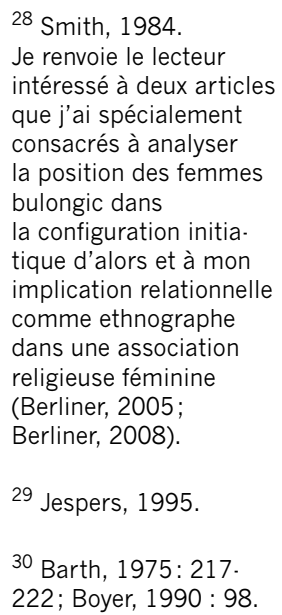


besoin de silence. Les initiés ne pouvaient publiquement parler de Mossolo Kombo et de Sangaran sans risquer gros. Il fallait savoir se taire, sans quoi un initié risquait de se faire égorger ou empoisonner, et les initiés eux-mêmes étaient soumis à de nombreuses interdictions. À la fin de l'initiation, les nouveaux circoncis, jusqu'alors maintenus dans l'état d'ignorance de non-initiés, découvraient que Mossolo Kombo et Sangaran étaient matérialisés par des objets sculptés, portés par les bilgbilifang, leurs initiateurs. Quelques jours avant leur retour au village, les jeunes initiés étaient d'ailleurs réunis pour que leur soit montré le système d'arrimage des masques. On leur enseignait également que les gémissements de Mossolo Kombo, reconnaissables entre tous, étaient produits à partir d'une corne d'animal et que les bruits effrayants de bèn bé kiyitun, entités marines accompagnant Mossolo Kombo, provenaient du fruit du papayer. Ces révélations progressives sur la nature du masque et ses artifices se déroulaient sous le sceau d'interdictions et de menaces de mort. Un vieil initié raconte qu'à la fin de l'initiation, "les vieux creusaient une fosse dans laquelle chaque initié devait uriner et cracher. Si un jeune se risquait à révéler le moindre secret, on allait le retrouver par son urine et sa salive et le tuer ». Pour tous les initiés, il était sous peine de mort formellement interdit de dire que Mossolo Kombo et Sangaran aient jamais pu être des objets sculptés : « Jamais tu ne peux dire le nom de celui qui sculpte " ou encore " jamais tu ne peux dire le nom de celui qui porte $»$

Autant dire que les précautions sociales et rituelles pour maintenir l'étanchéité du secret étaient omniprésentes. La sculpture de ces objets était d'ailleurs une opération très secrète, faite de nuit en pleine forêt. De même pour les opérations de nettoyage ou de destruction des masques, qui se faisaient dans la plus grande intimité. Lors des performances publiques, le rôle du porteur du masque, qui devait impérativement dissimuler son identité, était périlleux, et la moindre chute risquait de dévoiler les artifices de la simulation et de porter atteinte à l'édifice rituel dans son entièreté. Tous ces artifices, médiateurs de la fiction, supportaient un 
discours, celui des hommes initiés, qui présentait Mossolo Kombo et Sangaran comme de terrifiantes entités invisibles que seuls les vieux initiés pouvaient connaître. Inscrites au cœur d'un processus de simulation et de dissimulation ${ }^{31}$, les deux entités mettaient en jeu une configuration initiatique qui imposait des lignes de partage très strictes entre catégories sociales, savoirs, apprentissages, discours et lieux. Comme c'est le cas dans de nombreuses sociétés secrètes, ce sont précisément ces découpages et articulations sociales pluriels, déployant entre initiés et non-initiés une épistémologie du secret, qui permettaient au système des masques d'être efficace.

\section{Les jeunes Bulongic ne sont pas amnésiques}

1954 est l'année de la dernière circoncision bulongic. En 1955, le passage d'un expert coranique connu sous le nom d'Asékou Bokaré mit un terme définitif à ces pratiques rituelles ainsi qu'à la plupart des pratiques pré-islamiques ${ }^{32}$. Plus jamais, disent les vieux Monchonais, une initiation «bulongic " n'aurait lieu dans l'obscurité des forêts sacrées. Ni Mossolo Kombo ni Sangaran ne sortiraient jamais plus. Les tambours allaient se taire et les danses s'interrompre. La consommation de vin de palme devait cesser. Le calendrier rituel allait incorporer de nouvelles dates, et les circoncisions se fondre au rite musulman. Depuis 1955, à entendre mes interlocuteurs, une nouvelle société, fermement encadrée par l'islam, a vu le jour.

Nés après 1954, les jeunes Bulongic d'aujourd'hui n'ont pas été initiés, ils n'ont jamais vu danser les masques Mossolo Kombo et Sangaran. Ils n'ont rien connu de ce temps où leurs pères et grands-pères ne faisaient que «boire et danser ». Pourtant, ce passé pré-islamique est toujours saillant et valorisé, et les jeunes bien porteurs d'une mémoire religieuse. Ils ont entendu parler de l'initiation, de rites efficaces, de lieux secrets, d'interdictions, et ils véhiculent une série de stéréotypes relatifs à la " coutume». De Mossolo Kombo ${ }^{33}$, tous les jeunes adultes savent bien qu'il s'agit d'un génie, lié aux forêts sacrées, aux secrets, à l'initia-

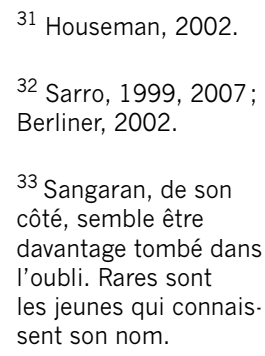


34 Tukoro se trouve sur le cordon dunaire au nord de Monchon. C'est de là que surgissaient jadis Mossolo Kombo et Sangaran Iorsqu'ils faisaient leur apparition de la mangrove vers le village. tion et à sa violence. Ils insistent sur le fait qu'il devait s'agir d'un génie "très puissant", qui pouvait se révéler "très dangereux " et suscitait la peur des non-initiés. D'ailleurs, s'exclame Abdou, 20 ans, "tout le monde avait très peur de Mossolo Kombo. Quand il criait, tout le monde était obligé de courir et de s'enfermer dans la maison, fenêtres fermées". Preuves de sa puissance: "quand Mossolo Kombo criait à Tukoro, même si tu étais à Monchon, tu l'entendais ", "il se déplaçait comme la lumière ", "il était haut comme les palmiers ", « il pouvait venir crier dans ton pantalon "... sont autant de phrases recueillies auprès des jeunes du village. Ces mêmes pouvoirs extraordinaires favorisaient la riziculture et aidaient les Bulongic à « se protéger contre leurs ennemis, quand il y avait les guerres entre les ethnies».

Certes, s'ils utilisent l'imparfait pour parler de l'initiation, c'est le présent qui domine les discussions des jeunes relatives à Mossolo Kombo. La plupart d'entre eux ignorent pourtant que le masque avait une composante matérielle, et que cette matérialité a été abolie lors de la venue d'Asékou Bokaré dans les années cinquante. Pour eux, Mossolo Kombo représente avant tout une puissance religieuse vraiment bulongic, douée d'autonomie et imprévisible, que " nos" vieux connaissent et qui inspire encore la terreur. Ainsi, Oumar, 18 ans, me disait que "Mossolo Kombo est là, avec femme et enfants, il vit à Tukoro ${ }^{34}$. On peut le voir là-bas en train de prier. Quand le soleil est chaud dans la plaine et que tu marches seul, tu peux le voir ". Abdoulaye, 19 ans, soulignait quant à lui que "Mossolo Kombo est à Tukoro. Il s'habille comme un vieux Peul mal foutu. Si tu le rencontres, il ne te regarde jamais dans les yeux. Il se promène un peu partout ", tandis qu'un autre remarquait que "quand on tire un coup de fusil, on peut le voir à Tukoro avec un turban». Lors de mon dernier séjour, un groupe de jeunes a raconté avoir vu le génie Mossolo Kombo, alors qu'ils s'apprêtaient, sans le savoir, à couper du bois dans une ancienne forêt sacrée. Ils m'ont raconté que le génie était apparu devant eux pour leur dire qu'il ne fallait pas couper le bois en ce lieu interdit. Et de nombreux jeunes 
de souligner que «tout le monde a encore peur de Mossolo Kombo, il tue encore beaucoup. Il est partout en pays bulongic. Tout est là ».

Les rumeurs vont d'ailleurs bon train. Les vieux initiés continueraient de pratiquer en cachette. S'il est, en effet, une évidence pour tous ces jeunes qui n'ont jamais été initiés, c'est qu'il existe " encore " des secrets bulongic détenus par ces vieux "qui connaissent" et "qui ont fait la forêt sacrée ". "Il y a ces molumil jusqu'à maintenant ", " jusqu'à maintenant, les vieux peuvent le faire ", "jusqu'à présent, ça existe ", "ils continuent Mossolo Kombo, mais ils se cachent " sont autant de phrases qui résument leur point de vue. Peu importe d'ailleurs que les forêts sacrées aient été rasées lors du passage d'Asékou, les vieux vont aujourd'hui à la Mecque, ce «lieu uniquement pour les vieux où les Hadj voient tous les secrets de là-bas ", m'indiquait Abdoulaye. À l'instar des non-initiés de jadis, ces jeunes ont une conscience aiguë de leur exclusion de ces savoirs barricadés et mettent l'accent sur la relation très spéciale qui existe entre leurs pères et ces secrets. Comme le soulignait Oumar, 17 ans, "il faut être un vieux pour pouvoir parler avec Mossolo Kombo. Nous, on sait pas exactement d'où il est sorti, les vieux savent, mais ils ne le disent pas. Mossolo Kombo, ce sont les vieux qui connaissent ".

Le recours au temps de la coutume (tèm o kutum) est constant chez les jeunes villageois. La plupart d'entre eux y voient un avant presque parfait, fait de paix et de prospérité. Un passé radieux et, dans le même temps, dangereux parce que les vieux initiés savaient se défendre contre le mal et les agressions extérieures (de leurs voisins susu notamment), grâce à leurs molumil. Incarnant toutes ces pratiques rituelles qui faisaient la force des Bulongic, les « secrets des vieux initiés " sont, pour les jeunes villageois, le symbole d'un temps tout à la fois idyllique et terrifiant. Surtout, comme on le verra, il s'agit pour eux d'un temps qui n'attend que d'être ressuscité, quoiqu'ils reconnaissent, paradoxalement, être en attente d'une transmission qui est désormais « impossible ». Bien loin d'être amnésiques, confrontés à un présent difficile et pétri de frustrations, les jeunes semblent, au con- 
${ }^{35}$ Nora, 1977 : 227.

${ }^{36}$ Borofsky, 1987 :

97.100. traire, encombrés « jusqu'à la paralysie par une mémoire fascinée $^{35}$." Une mémoire où se mêlent fascination et crainte face à l'existence de ces dangereux secrets.

\section{Sécréter}

Tous ces propos montrent comment les jeunes adultes se sont évertués, sur les quelques bribes d'informations concédées par leurs taciturnes aînés, à construire la figure d'un monde qu'ils n'ont pas connu (mais sur lequel, croient-ils, les vieux en savent beaucoup plus que le peu qu'ils leur en ont dit). Ce sentiment ambigu de la présence de l'absent, de l'existence de quelque chose de dangereux et précieux, possédé par les seuls vieux initiés mais qui demeure caché et souterrain, je l'ai aussi ressenti. Autant les jeunes sont prolixes, autant leurs pères sont hermétiques, d'un accès difficile. Avec eux, j'ai d'abord rencontré le silence. L'on touche ici à un domaine d'une extrême sensibilité, saturé d'interdictions. Combien de vieux initiés approchés pour s'entendre dire, avec un sourire aux lèvres : «La coutume? On ne peut pas en parler, on a juré de ne rien dire... "Très vite, je me suis distingué par ma maladresse : en pays bulongic, on ne pose jamais de questions directes relatives aux secrets des vieux initiés. L'imprudente curiosité pourrait être punie de mort, comme si la parole était lourde d'un danger dont seul le silence peut préserver. "Avant, même si tu parlais à un palmier de Mossolo Kombo, celui-là risquait de te dénoncer. Une mouche pouvait te dénoncer ", dit-on aujourd'hui encore à Monchon.

De fait, parler avec un vieil initié est un art, les jeunes bulongic vous le diront. Pour s'entretenir avec lui, il faut savoir choisir la bonne heure, l'endroit adapté, "frotter " le vieux avec de la kola ou du vin de palme, poser peu de questions, se satisfaire de peu, revenir souvent et, puis, un jour, « le vieux va peut-être se mettre à parler ». S'asseoir à côté d'un initié est une souffrance, faite de subordination et d'humiliation ${ }^{36}$. La curiosité intrusive de l'ethnologue est toujours malvenue et, à chaque entretien, un scénario quasi similaire se répète. Aborder le sujet du passé initia- 
tique déclenche de la part des vieux de Monchon soit un refus pur et simple de commenter : " ce sont des choses que l'on ne peut pas dire ", soit un " rire de rupture " qui met fin à cette séquence précise de l'interaction, soit un silence qui en dit long. Bref, la communication ne "prend" pas. Seuls ces regard furtifs, ces soupirs, ces sourires, ces airs, ces manières, ces attitudes graves lors de nos premières rencontres, ces formules lapidaires et ces descriptions stéréotypées durant les entretiens (du genre "ici, il n'y a plus rien. On a tout abandonné de la coutume. ") ou encore ces absences remarquées à nos rendez-vous signalaient la "sécrétion ${ }^{37}$ " à l'œuvre, comme autant d'indices de la fondamentale présence des secrets. La question est alors : "Comment est-ce qu'on n'en parle pas? ${ }^{38}$ "

Pleines de silences et de sous-entendus, ces conversations avec les vieux initiés qui se sont tissées au fil des mois de ma recherche ont pourtant été particulièrement édifiantes. Ils ne cessaient de répéter que l'abandon des pratiques pré-islamiques, de l'initiation et des forêts sacrées avait été une chose nécessaire. En reprenant la rhétorique de l'islam et celle de Sékou Touré, ils se répandaient en jugements moraux sur les pratiques dites «animistes». La venue de la religion était toujours narrée sur le mode apologétique. Soignant leur image de musulmans respectables, leurs récits décrivaient la persévérance, la passion, l'audace et la témérité des premiers convertis. En 1954, les gens étaient devenus "fatigués de la coutume " parce que "les fétiches ne faisaient pas avancer. La coutume, cela ne faisait pas progresser ». À en croire ces vieux, en ces années 1950 où ils avaient vingt ans et où la Guinée s'acheminait vers l'indépendance, les pratiques rituelles étaient devenues incompatibles avec les exigences de l'heure. Beaucoup insistaient sur leur violence « inutile». Combien de fois les ai-je entendu remarquer que "la coutume, cela ne faisait que tuer les gens. La jeunesse était contre les fétiches. Elle voyait les pertes que cela causait. On a décidé d'enterrer la coutume ».

Pourtant, il y a dans le rapport que les vieux Bulongic entretiennent vis-à-vis de la coutume quelque chose d'am-

37 Zempléni, 1976 : 320.

38 Williams, $1993: 7$. 
${ }^{39}$ Tuzin, 1997.

${ }^{40}$ Chez les Kpelle, cf. Bellman, 1984; chez les Fang du Cameroun, cf. Boyer, 1990.

${ }^{41}$ Sarro, 2002. bivalent. Une sorte de dissonance cognitive et morale, qui serait caractéristique de l'effondrement violent et cathartique des systèmes religieux de ce type ${ }^{39}$. Dans le même temps qu'ils insistent sur la nécessité des changements qui se sont produits jusqu'en 1954, ces vieillards décrivent aussi avec nostalgie l'époque de l'initiation et des rituels pré-islamiques comme une sorte d'âge d'or, de passé idyllique, tout en mettant l'accent sur la perte réelle de puissance rituelle qu'a occasionnée la fin de cette époque. La disparition de l'initiation est aussi, pour beaucoup, présentée comme un affaiblissement de la vitalité rituelle du pays bulongic. Et les subtiles références à ce passé, où ils étaient de "vrais " Bulongic, dotés de secrets qui confèrent à ceux qui les détiennent une malignité et une efficacité rituelle extraordinaires, abondent : " au temps de la coutume, il y avait des vrais hommes ", "la forêt sacrée, c'était fort ", "avant, grâce à la forêt sacrée, les Bulongic étaient les plus malins du monde ». Dans la forêt sacrée, disent certains d'entre eux, « on parlait et on entendait tout. C'était la force là-bas. Il est dommage qu'un Ilongic [sg. de Bulongic] ne fasse pas la forêt ".

Au fil des entretiens, les secrets révélés dans la forêt sacrée m'étaient décrits comme un savoir efficace, potentiellement dangereux (puisqu'il pouvait « tuer»), et qu'il ne fallait en aucun cas divulguer. Et surtout, ce savoir devait être littéralement incorporé : "on mangeait les secrets en forêt sacrée " ou " les circoncis mangeaient la forêt » sont le genre de formules utilisées par les vieux initiés. Ces expressions mettent en avant l'idée d'un savoir-substance mangé par les initiés lors de la réclusion initiatique, comme c'est le cas dans de nombreuses populations d'Afrique occidentale et centrale $^{40}$. Ainsi, lors de la circoncision chez les Baga Sitem, les secrets sont "mangés " par les initiés, ce qui suppose bien plus que la transmission explicite d'une connaissance secrète, mais implique surtout l'incorporation des secrets dans la chair même des initiés ${ }^{41}$. Pour acquérir ce savoir dangereux, il fallait avoir enduré la violence initiatique. En témoignent les cicatrices de l'initiation que ces vieillards m'exhibaient avec fierté lors de nos interviews. 
C'est la même ambivalence, entre nostalgie pour le temps de la coutume et nécessité d'y avoir mis fin, qui surgit lorsqu'il est question de Mossolo Kombo, l'entité initiatique qui jouait un rôle crucial dans le paysage rituel d'avant 1954. Quand je montrais des photographies de l'objet bansonyi (l'objet matériel a été un médiateur dans mon rapport aux vieux initiés), les vieux initiés de 1937 et de 1954, qui ont jadis connu les performances de Mossolo Kombo, ont toujours réagi de manière stéréotypée. D'abord, ils se sont étonnés que j'ai pu obtenir pareille photographie et ont strictement refusé de parler de ces choses surtout devant femmes et enfants. Ensuite, ils s'exclamaient "Mossolo... ", souriaient, se mettaient à chanter et à parler avec nostalgie. Tous ont été particulièrement discrets à ce sujet. Ni avant 1954, ni après, les paroles relatives à Mossolo Kombo n'ont jamais sous peine de mort porté sur l'objet lui-même. Aujourd'hui encore, il est toujours difficile de laisser entendre en présence de non-initiés que le masque circonciseur a pu être, en partie, un artefact en bois. Cependant, les vieux Monchonais me confiaient en privé qu'il s'agissait effectivement d'un bois sculpté : "Ça sur la photo, ce n'est qu'un bois, c'est nous qui l'avons sculpté... » Ceux qui acceptaient de me parler reconnaissaient qu'il n'existait plus depuis 1954 de sculpteurs pour le tailler, ni de grandes initiations collectives ou de danses publiques pour mettre en scène ses apparitions.

Pourtant, dans le même temps qu'ils reconnaissaient discrètement la part de matérialité du masque, tous les vieux initiés s'accordaient pour dire " qu'il y avait quelque chose à côté du bois ». Mossolo Kombo, c'était secrètement un bois sculpté et détruit par les hommes, mais c'était avant tout le génie représenté par ce bois. Le discours des vieux initiés présente toujours Mossolo Kombo comme un génie que l'on trouve dans la mer et dans la mangrove. Et le génie, qui est un génie marin, n'a, quant à lui, pas disparu :

Les masques disparaissent, mais les génies restent. Mossolo Kombo, il existe encore. On peut le voir quand on est malin. Il n'a pas disparu. On n'a plus le bois, mais un génie ne meurt pas. Il y a des gens qui recourent encore secrètement à lui. 
Les bois sont brûlés, mais les formules [rituelles] sont dans les poches.

Les vieux initiés sont toujours laconiques à ce sujet, mais verbalisent suffisamment pour mettre en scène la présence de Mossolo Kombo, en tant que puissance religieuse qu'eux seuls connaissent, du fait de leur passage dans la forêt sacrée au temps de la coutume. Comme le souligne un vieux de Monchon :

Mossolo est là, les molumil sont là. Les gens sont là. Quand les enfants disent que Mossolo Kombo est là, ils ont raison. Moi-même, je te dirais qu'il est là. Mossolo Kombo, ce sont des hommes et un génie. Nous pouvons le faire demain matin. Il est là parce que nous sommes là. Mais quand nous allons partir...

\section{Une impossible transmission}

"Mais quand nous allons partir..." est un lamento sur l'impossibilité de la transmission maintes fois répété à l'oreille de l'anthropologue. Les jeunes hommes du village ont pourtant soif de transmission. Ils invoquent la perte d'un savoir qui permettraient aux Bulongic d'être mieux armés contre les aléas de la modernité, et souhaiteraient avoir accès à ces secrets qui leur permettraient d'être à nouveau " les plus malins du monde ». Les jeunes de Monchon se sont d'ailleurs réunis pour aller demander aux vieux " les secrets de Mossolo Kombo ». "La perte, souligne Mady, ce n'est pas bon pour nous, les générations montantes. Parce que certaines choses se manifestent et que si l'on avait ses secrets, cela n'allait pas se faire. À l'époque de Mossolo Kombo, les autres ethnies n'osaient pas se moquer de nous. Il faudrait revenir à la coutume. " Mais les jeunes ont peur aussi. C'est là toute l'ambivalence de ces secrets, convoités mais terrifiants. Comme le souligne Abdoulaye, « les jeunes ont peur de savoir, même une mouche peut te trahir. Un jeune a voulu savoir, ils l'ont tué. Voilà pourquoi les jeunes ont peur de demander aux vieux ».

De leur côté, face à la pression des jeunes qui invoquent la perte de savoir, les vieux insistent sur le fait qu'il n'y a plus de transmission possible et énumèrent les obs- 
tacles sociaux et culturels à la transmission aujourd'hui. Avec la disparition des initiations, disent-ils, il n'y a plus de contexte pour transmettre dans la souffrance cette connaissance qui permettait aux Bulongic "d'être les plus forts". Parce qu'ils ont été acquis dans un contexte rituel tout à fait particulier, au temps perdu de la coutume, ces savoirs sont aujourd'hui intransmissibles. Et un vieil homme de souligner que "nos pères ont vécu la forêt sacrée. Après, une petite partie a été donnée à nos grands frères. Nous, on a eu un peu, mais nous sommes les derniers Bulongic ". Refusant de transmettre, les vieux initiés invoquent, sur un ton fataliste, la dégénérescence des jeunes générations bulongic :

Les jeunes sont mal élevés et ne savent pas garder le secret. Ils vont mal utiliser les secrets. Qui serait capable de reprendre les secrets aujourd'hui? À notre époque, on avait reçu une bonne éducation dans la forêt.

Les interactions entre les jeunes adultes, persuadés qu'il y a quelque chose à savoir, et les vieux initiés sont toujours marquées par la distance. Sur le plan de la communication intergénérationnelle, c'est l'étanchéité, la méfiance qui prévalent aujourd'hui. Les jeunes soulignent que «les vieux ne discutent pas avec les jeunes des molumil. Il n'existe pas de causerie entre les vieux et les jeunes". Pourtant, leurs interactions se caractérisent aussi par une étrange congruence. Je ne compte pas les entretiens menés avec des jeunes, soudainement interrompus par un vieil initié qui venait y monopoliser la parole et imposer le silence aux jeunes par un «eux, ils ne connaissent rien, ce sont des enfants. La forêt sacrée, c'est nous ». Et les jeunes d'acquiescer : "Oui, nous on ne connaît rien. On n'a pas fait la forêt. Lui, il connaît! » Et, non contents de reconnaître leur exclusion et leur ignorance de ces savoirs barricadés ("Nous, nous ne connaissons pas les secrets d'avant»), les jeunes villageois sont aussi les premiers à admettre leur responsabilité dans cette impossible transmission. Avec gêne, ils admettent, sans contester, qu'ils sont "de petits Susu ", se décrivant la plupart du temps comme "incapables", " vantards ", « égoïstes ", " irrespectueux ", «mal élevés " et 
« ignorants ", bref tous ces défauts qui les rendent a priori " incapables de reprendre les vieilles histoires " :

Les vieux n'acceptent pas de nous donner les secrets. Ils ne font pas confiance dans le comportement des jeunes. C'est vrai, les jeunes font facilement du mal. C'est pourquoi, les vieux ont refusé de transmettre. Ils ont peur que nous allions divulguer les secrets à l'extérieur.

À entendre les jeunes Bulongic exprimer leur exclusion explicite de ces savoirs barricadés et faire leurs les dépréciations émises par les vieux initiés, je retrouve l'exemple des Samburu décrits par Paul Spencer où, sans cesse convaincus par les vieux de leur immaturité, les jeunes admettent que " tous ce dont les vieux les accusent est parfaitement vrai ${ }^{42}$ (all the elders accuse them is quite true)».

\section{Le pouvoir mystificateur des vieux initiés}

Parmi les critères qui permettent aux vieux, les atemil, de se distinguer entre eux et de jouer de leur autorité figure l'expérience, indépassable, de la forêt sacrée. La plupart des atemil de Monchon ont été circoncis en forêt sacrée, celle de Mossolo Kombo ou de Sangaran, en 1937 ou en 1954. Depuis 1954, les pratiques initiatiques ont disparu, ce qui a entraîné une stagnation des classifications rituelles. Pourtant, bien que la machine classificatoire se soit en quelque sorte arrêtée net, les grades d'antan restent opératoires dans la société bulongic d'aujourd'hui. À ce jour, tous les anciens initiés de Monchon sont restés soit des bilgbili, soit des bilgbilifang, tandis que la plupart des circoncis d'avant 1937, qui furent les bilgbilifang des circoncis de 1937, sont morts. Les classifications rituelles distinguant les bilgbili et bilgbilifang de l'année 1954 sont restées en vigueur depuis cette date. Les bilgbili continuent de respecter leurs bilgbilifang. Mon interprète, circoncis en 1954, m'a exprimé à plusieurs reprises sa difficulté à poser certaines de mes questions qui pourraient offenser ses bilgbilifang. Lors de réunions d'atemil, les hommes continuent de se regrouper hiérarchiquement par classe d'âge d'initiés (" ceux de 37 », « ceux de 54 »). Le fait d'avoir été impliqués activement 
dans le fonctionnement de l'institution initiatique, comme les initiés de 1937 lors de la circoncision de 1954, constitue un critère de distinction important en matière de connaissance rituelle pré-islamique : par exemple, tel vieux en sait beaucoup "parce qu'il fut le premier des circoncis et a dû faire amancong wala». Avoir été circoncis en 1937 suppose a priori que l'on en sache plus qu'un circoncis de 1954.

Non initiées aux secrets bulongic, les jeunes générations d'après 1954 ne sont, quant à elles, plus intégrées dans une telle classification. Pour les vieux initiés, ces jeunes qui "viennent de naître et ne connaissent rien " occupent la position de non-initiés. Mais en l'absence d'initiation et donc de transmission des secrets, il n'y a plus aujourd'hui de catégorie " dépositaire ". Seuls persistent les vieux initiés, avec leurs secrets, et tous les autres non-initiés, qui, par leur aveu d'ignorance et leur crainte, contribuent à pérenniser la structure du secret. Comme jadis les non-initiés, les jeunes ignorent la part de simulation que comportent les secrets de leurs aînés : s'ils savent bien des choses sur le passé fascinant et dangereux de leurs parents, ils ne savent rien des trucs et des illusions cachées qui contribuaient autrefois au bon fonctionnement de la simulation initiatique. Alors que l'initiation supposait autrefois la révélation progressive de certains de ces trucs au cours d'épreuves douloureuses dont il n'y a plus d'équivalent, les jeunes d'aujourd'hui resteront probablement avec leur connaissance de non-initiés, du moins tant que perdurera le silence des vieux initiés.

À Monchon, entre les initiés et leurs fils, les références explicites à la coutume et aux molumil sont en effet très rares. $\grave{A}$ en croire mes interlocuteurs, elles se produisent très occasionnellement dans l'intimité des interactions entre pères et fils. Il ne m'a pas été donné d'assister à de telles formes d'interaction, rarissimes et secrètes. Par contre, comme j'ai pu le remarquer à plusieurs reprises, certains contextes publics peuvent donner lieu à des allusions voilées à la coutume et aux molumil. Dans ces situations, pour les vieux initiés, mentionner l'initiation, Mossolo Kombo ou la force des anciens est loin d'être une simple figure de rhétorique entretenue par nostalgie. De fait, ces références 
voilées servent la plupart du temps une intention particulière, à savoir celle de dénigrer le comportement des jeunes adultes et de les intimider en leur signifiant l'existence d'expériences liées aux molumil qu'ils n'ont pas vécues et dont ils sont à jamais exclus. Par exemple, je me souviens de ce vieil homme qui, furieux à l'endroit de ses jeunes garçons, leur cria : "Ne vous moquez pas de moi! Vous ne me connaissez pas! Moi, je suis Mossolo Kombo! » Dans une situation similaire, pointant du doigt ses fils, un vieillard s'exclama : "Les jeunes, ils ne connaissent rien. Ils sont des Susu aujourd'hui. Jadis, on avait une bonne éducation dans la forêt sacrée. Mais eux, ils ne connaissent rien. Nous sommes les derniers Bulongic. "

Cette formule, "nous sommes les derniers Bulongic", maintes fois entendue lors de ces interactions, est particulièrement intéressante à analyser. Elle doit être pensée dans le tissu même de cette configuration de relations toute spécifique qui existe entre les vieillards et les jeunes gens. En effet, jamais un Ilongic ne prononcera, avec sérieux, une telle expression devant un non-Ilongic (un Susu ou un Peul). Au contraire, s'identifier comme Ilongic (ou Baga) dans un environnement non-Ilongic est une source de prestige, tant l'image de populations littorales saturées de secrets a la peau dure en Guinée. Pour un vieil homme, en revanche, s'exclamer devant ses fils : " nous sommes les derniers Bulongic » ou " nos enfants sont des Susu » est une manière de leur signifier qu'ils sont exclus d'un savoir dont les vieux sont toujours détenteurs. « Nous sommes tous des Bulongic. Mais ceux qui n'ont pas fait la forêt ne peuvent pas connaitre comme nous ", me rappelait un vieil initié. En faisant référence aux expériences initiatiques liées aux molumil, expériences charnelles exclusivement vécues dans les forêts sacrées de Monchon, les vieux initiés entretiennent l'idée qu'il existe un ordre supérieur de vérité auquel les jeunes n'ont pas part. De fait, comme je l'ai dit plus haut, au fil des discussions, j'ai eu l'impression que les vieux initiés faisaient tout pour manifester qu'il existe encore du secret, tout en insistant sur le caractère très dangereux et précieux de ces secrets acquis au temps de la coutume, mais 
aussi sur leur impossible transmission aujourd'hui. En fait, le discours que les vieux tiennent sur leur passé religieux est saturé de significations métacommunicationnelles et fait partie de la fabrication du secret. À grands traits, son contenu métacommunicationnel est : "Tout est là, c'est dangereux et cela produit des effets rituels extraordinaires, mais on ne peut pas vous le transmettre."

Parce qu'il est le discours des vieux initiés, ce discours est "vrai ", au sens qu'il inspire du respect et, souvent, de la terreur. Réputés avoir côtoyé en forêt sacrée des entités initiatiques, les vieux initiés sont ces "personnes faites sur mesure " (customised persons) décrites par Boyer ${ }^{43}$. Détenteurs de la vérité, ils sont aussi ces maitres de l'illusion qui reconnaîtront, dans certains contextes d'interlocution, que les performances de Mossolo Kombo, bien que mettant en scène une entité invisible, étaient aussi faites de mensonges, d'illusions et de trucages. L'on sait que le système initiatique met en jeu des instances d'intimidation, de mystification et de manipulation coercitive de la part des vieux initiés ${ }^{44}$. Dans l'intimité des entretiens, les vieux Monchonais reconnaissent leur capacité à mystifier les jeunes :

Mossolo Kombo et Sangaran, je ne dirai jamais ce que c'est devant une femme. Jamais une femme ne peut savoir. Sinon les gens qui ont peur de vous n'auront plus peur.

Ils savent ce que veut dire fiction initiatique (simuler et dissimuler) et, bien que discrets, signalent qu'il s'agit de leurs propres artifices :

Quand Asékou Bokaré est venu pour détruire les fétiches, on a raconté aux femmes et aux enfants qu'on avait apporté les génies à la mer. C'est pour cela que tu entendras des enfants te dire que Mossolo Kombo est à la mer.

Comme le souligne Tuzin, chez les Arapesh, «by this time men have reached this grade of the technique of manipulating - unconciously or by design - truth and illusion to their own advantage ${ }^{45}$. "À la question de savoir pourquoi le culte de Tambaran n'est pas ouvert aux femmes, les interlocuteurs de Tuzin remarquent que "it is true that sometimes men feel ashamed and guilty [...]. But if we told them now that for all

${ }^{43}$ Boyer, 1990 : 94-106.

${ }^{44}$ Houseman, 1993.

${ }^{45}$ Tuzin, $1980: 283$. 
46 Ibid, p. 302.

47 Ibidem.

${ }^{48}$ Bellman, 1984 : 140.

${ }^{49}$ Bastide, 1970 : 92. these generations they had been deceived, they would make life unbearable for us. There is nothing we can $\mathrm{d}^{46}$. " Et l'auteur de conclure que " the dogma, then, cannot be relaxed, but must be ever renewed ${ }^{47}$ ". À Monchon aussi, les vieux initiés sont de talentueux producteurs d'indices du secret et, tandis qu'ils ont cessé de simuler la présence de leurs entités en manipulant masques et autres objets rituels, ils continuent de jouer la fiction initiatique.

\section{Conclusion}

Parlant de cette célèbre société initiatique que l'on retrouve depuis le sud de la Guinée jusqu'au Libéria, Bellman remarque que " the Poro as an institution has endured for centuries. It has been able to do so not because of the secrets that it protects but because it is the very embodiment of the procedures necessary for "doing" secrecy. The society's rituals are an expression of the form of secrecy rather than its content. The society has survived because of its concern with form ${ }^{48}$. "À Monchon, sous le bougé dynamique de la vie quotidienne, persiste, liée au passé pré-islamique, une forme interactive qui repose sur la distinction entre "initié " et "non-initié », entre ces vieux initiés "qui savent", ultimes détenteurs de ces précieux secrets, faits de cicatrices et de violence initiatique et qu'ils se refusent à transmettre, et de jeunes jamais initiés auxquels rien ne pourra être transmis. "Mémoire d'un système de rapports entre les individus ${ }^{49}$ ", cette forme, bien qu'aujourd'hui en dehors de tout rituel d'initiation, continue d'organiser entre elles les attitudes et discours de différentes catégories sociales. Certes, le passé religieux d'avant l'islamisation continue d'habiter le présent de Monchon. Mais c'est à travers le prisme d'une forme interactive héritée qu'il continue de produire ses effets. Dans ces cultures du secret, il ne faut pas grand chose pour induire la peur et la fascination: des bribes de paroles, des attitudes nonverbales, des manières, le langage d'un corps, qui tous participent de la manifestation du pouvoir. 


\section{Références bibliographiques}

Adler, A.

1998 «Des rois et des masques. Essai d'analyse comparative (Moundang du Tchad, Bushong de l'ex-Zaïre)», L'Homme 145, pp. 169-203.

Barth, F.

1975 Ritual and knowledge among the Baktaman of New Guinea, Oslo/Yale, Universiteitforlaget/Yale University Press.

Bastide, R.

1970 "Mémoire collective et sociologie du bricolage », L'Année Sociologique XXI, pp. 65-108.

Bellman, B.

1984 The Language of secrecy: symbols and metaphors in poro ritual, New Brunswick, NJ, Rutgers University Press.

Berliner, D.

2002 Nous sommes les derniers Bulongic. Sur une impossible transmission dans une société d'Afrique de l'Ouest (Guinée-Conakry), Bruxelles, Université Libre de Bruxelles, thèse de doctorat, non publiée.

2004 "(Re) Discovering Landuma masks (Boké, Guinea-Conakry)», Arts et Cultures 5, pp. 134-143.

2005 "La féminisation de la coutume. Femmes possédées et transmission religieuse en pays bulongic (GuinéeConakry) », Cahiers d'Études Africaines 177, 45 (1), pp. 15-38.

2008 "The anthropologist in the middle of a tug-of-war (Guinea-Conakry)", Men and Masculinities 11, pp. 174-185.

Berliner, D. \& R. Sarro (dir.)

2007 Learning religion : anthropological approaches, Oxford, New York, Berghahn Books.

Bloch, M.

1998 How we think they think. Anthropological approach to cognition, memory and literacy, Boulder, Westview Press.

Bonhomme, J.

2006 " "La feuille sur la langue". Pragmatique du secret initiatique ", Cahiers gabonais d'Anthropologie 17, pp. 1938-1953.

Borofsky, R.

1987 Making history. Pukapukan and anthropological construction of knowledge, Cambridge, Cambridge University Press.

Boyer, P.

1990 Tradition as truth and communication. A cognitive description of traditional discourse, Cambridge, Cambridge University Press.

1997 La Religion comme phénomène naturel, Paris, Bayard Éditions.

2001 Et l'homme créa les dieux. Comment expliquer la religion, Paris, Éditions Robert Laffont. 
Coquet, $\mathrm{M}$.

1999 "Des objets et leurs musées ", Journal des Africanistes 69 (1), pp. 9-27.

Curtis, M.-Y.

1996 L'Art nalu, l'art baga de Guinée. Approches comparatives, Paris, Université

Paris-I, thèse de doctorat, non-publiée.

Debray, R.

1997 Transmettre, Paris, Éditions Odile Jacob.

Herdt, G.

2003 Secrecy and cultural reality. Utopian ideologies of the New Guinea men's house, Ann Arbor, University of Michigan Press.

Hojbjerg, C. K.

2002 «Inner iconoclasm. Forms of reflexivity in Loma rituals of sacrifice ", Social Anthropology 10 (1), pp. 57-75.

Houseman, M.

1993 "The interactive basis of ritual effectiveness in a male initiation rite ", in P. Boyer (dir.), Cognitive Aspects of Religious Symbolism, Cambridge, Cambridge University Press, pp. 207-224.

2002 "Dissimulation and simulation as forms of religious reflexivity", Social Anthropology, 10(1) : 77-89.

2004 «Vers une psychologie de la pratique rituelle? ", Critique 680-681, pp. $102-$ 114.

Houseman, M. \& C. Severi

1994 Naven ou le donner à voir. Essai d'interprétation de l'action rituelle, Paris, CNRS-Éditions/Éditions de la Maison des Sciences de l'Homme.

Jespers, $\mathrm{P}$.

1995 "Le masque et la parole (Minyanka, Mali) ", in L. de Heusch (dir.), ObjetsSignes d'Afrique, Tervuren, Snoeck-Ducaju en Zoon, pp. 37-57.

La Fontaine, J.

1985 Initiation. Ritual drama and secret knowledge across the world, New York, Penguin Books.

Lamp, F.

1996 Art of the Baga. A drama of cultural reinvention, New York/Munich, The Museum for African Art/Prestel Verlag.

McCauley, R. N. \& T. Lawson (dir.)

2002 Bringing ritual to mind : psychological foundations of cultural forms, Cambridge, Cambridge University Press.

Nora, P.

1977 «Mémoire de l'historien, mémoire de l'histoire. Entretien avec J.-B. Pontalis ", Nouvelle Revue de Psychanalyse 15, pp. 221-232. 
Paulme, D.

1956 "Structures sociales en pays Baga », Bulletin de l'IFAN 18, série B, pp. 98-116.

1957 "Des riziculteurs africains », Cahiers d'Outre-Mer 10, pp. 257-278.

1958 "La notion de sorcier chez les Baga", Bulletin de l'IFAN 20, série B, pp. 406-416.

Sarro, R.

1999 Baga identity : religious movements and political transformations in the Republic of Guinea, Londres, University College, thèse de doctorat, non-publiée.

2002 "The iconoclast meal : destroying objects and eating secrets among the Baga of Guinea ", in B. Latour \& P. Weibel (dir.), Iconoclash, Cambridge (Mass.), MIT Press, pp. 227-230.

2007 "Hermetic huts and modern State : the politics of iconoclasm in West Africa », in S. Boldrick \& R. Clay (dir.), Iconoclasm : contested objects, contested terms, Aldershot, Ashgate, pp. 261-277.

Shaw, R.

1997 "Secret Society ", in J. Middleton, (dir.), Encyclopedia of Africa south to the Sahara, New York, Simon and Schuster Macmillan, pp. 44-47.

Simmel, G.

1996 Secret et sociétés secrètes, Aubenas, Circé Poche.

Sinayoko, S.

1937 "Quelques coutumes "Baga-Foré” ", L'Éducation africaine 98 (oct.-déc.), pp. 220-225.

Smith, P.

1984 "Le "mystère" et ses masques chez les Bedik ", L’Homme 24 (3-4), pp. 5-33.

Soual, P.

2003 "L'intransmissible ", in J.-N. Dumont (dir.), Transmettre. Quel est le prix de nos ruptures, Lyon, Éditions de l'Emmanuel, pp. 99-113.

Spencer, P.

1962 The Samburu. A study of gerontocraty in a nomadic tribe, Londres, Routledge and Kegan Paul.

Tuzin, D.

1980 The Voice of Tambaran. Truth and illusion in Ilahita Arapesh Religion, Berkeley/ Los Angeles/Londres, University of California Press.

1997 The Cassowary's revenge. The life and death of masculinity in a New Guinea Society, Chicago/Londres, The University of Chicago Press.

Whitehouse, H. (dir.)

2001 The Debated mind. Evolutionary psychology versus ethnography, Oxford, Berg.

Williams, P.

1993 Nous, on n'en parle pas. Les vivants et les morts chez les Manouches, Paris, Éditions de la Maison des Sciences de l'Homme.

Zempléni, A.

1976 "La chaîne du secret ", Nouvelle Revue de psychanalyse 14, pp. 313-325.

1996 "Savoir taire. Du secret et de l'intrusion ethnologique dans la vie des autres ", Gradhiva 20, pp. 23-41. 